\title{
A Novel Switching Control for Induction Motors Using a Robust Hybrid Controller that Combines Sliding Mode with PI Anti-Windup
}

\author{
Bilel Aichi1*, Khedidja Kendouci \\ ${ }^{1}$ Group of Control, Laboratory of Electrical Drives Development (LDEE), Department of Electrical Engineering, University of \\ Science and Technology of Oran Mohamed Boudiaf, P. O. B. 1505, El-Mnaouar, 31000 Bir El Djir, Oran, Algeria \\ * Corresponding author, e-mail: bilel.aichi@univ-usto.dz
}

Received: 30 January 2020, Accepted: 15 April 2020, Published online: 06 October 2020

\begin{abstract}
Induction Motors are the most used machines in the industrial field since the last century, due to their low cost, high robustness with satisfactory performance. However, they are still difficult to control compared to the DC motor because of the non-linearity presented in the mathematical model. Sliding mode theory is often used to develop powerful control against various internal and external disturbances. However, the chattering problem caused by the attractive part of the regulator is a serious problem for its applications. In this paper, the proposed solution for having an optimal performance consists in combining the Sliding Mode Control with an anti-windup proportional-integral regulator. This is achieved through a simple linear supervisor that can activate the sliding mode at start-up and transient regimes while making the second controller drive the steady state. The asymptotic stability of the delivered control signal is ensured via the Lyapunov method. This allows us to benefit from the advantages of the two regulators without having their disadvantages. This new hybrid technique can potentially offer very promising results in terms of robustness and control efficiency. The validation of this theory was carried out by simulation and then by practical implementation using a dSPACE-DS-1104 control board. The obtained results show a high-performance control with very good robustness against parametric variations and remarkable stability during all different operating zones.
\end{abstract}

Keywords

switching control, Induction Motor (IM), regulation supervisor, PI Anti-Windup (PIAW), Sliding Mode Control (SMC), hybrid regulator

\section{Introduction}

With improvements in the field of power electronics and digital processors, the development of a powerful variable speed drive for Induction Motors (IM) became possible. However, it requires dealing with problems related to the non-linearity in the mathematical model, which is mainly due to the internal coupling between the states variables, and the parametric variations affected by thermal and magnetic factors. Several nonlinear techniques have been developed to achieve high-performance control such as neural and fuzzy control $[1,2]$, Backstepping techniques [3-5], adaptive methods [6, 7], strategies based on DTC [8] and so on. On the other hand, the Sliding Mode Control (SMC) has become a very interesting method, thanks to its simplicity and its robustness against various disturbances and parametric uncertainties. It is based on the development of a control law composed of two terms capable of forcing the error regulation to converge towards its equilibrium point, so that this law complies with the Lyapunov condition $[9,10]$. However, the problem of the attractive term that is specially made up by the "Sign" function can generate very high-frequency disturbances in the control signals. This is known as the chattering phenomenon. Several techniques have been developed to mitigate this obstacle. In [11], the researchers used the Supertwisting algorithm which is considered among the best method to eliminate the chattering phenomenon and to have a good performance. The authors of [12] present a new sliding mode design which uses an adaptive switching gain with an integrator. This approach can guarantee a good dynamic response; in addition, the chattering can be reduced significantly. In the last few years, hybrid control techniques that combine sliding mode with other techniques have received much attention in the nonlinear system control field. The main objective is to exploit the advantages 
of both controllers. The work by [13] presents a combination of Fuzzy-Sliding-mode with a nonlinear controller based on Lyapunov's theorem. The switching control was ensured through a two-dimensional Gaussian supervisor. The obtained results show a total elimination of chattering with good control dynamics; however, the computing load was increased. The study by [14] explains a control technique using SMC with $\mathrm{H} \infty$ intended for the nonlinear systems control. Authors of [15] integrate a hybrid regulator in the vector control. The regulator uses SMC for transient regimes and Fuzzy-PI controls the steady state. In [16], the property of artificial intelligence was introduced to the general structure of SMC in order to obtain a high performance with better control signals. In [17], a hybrid controller structure was introduced and applied to nonlinear systems; the authors used SMC and a PI regulator, where a fuzzy supervisor was implemented to activate each controller in its operating zoon.

\subsection{Problems statement}

The simplicity of the PI regulator makes it one of the most commonly used in the industrial field. Its principle is based on a proportional action and another integral. This structure offers stable control signals recommended for the generation of control voltages in electrical drive systems. However, these performances remain modest for applications that require a fast dynamic response. On the other hand, The SMC offers a fast dynamic response with low sensitivity to parametric variations. Its simple structure is composed of an attractive command and another stabilizer. The control law modeling requires the information on external disturbances which can be very difficult to measure. Moreover, it can complicate the general structure. Similarly, their estimation can increase the algorithm complicity because it needs an important calculation time. In the absence of this information, SMC can offer good control only by using large gains. This condition can make it difficult to minimize the chattering phenomenon, which remains the major obstacle for real systems applications.

Efficiency and simplicity are the main objectives of our study which presents a practical combination between SMC and PI Anti-Windup (PIAW) in order to develop a nonlinear hybrid control method for the IM systems drive. The essential contribution of this paper is the development of a new powerful control scheme that enjoys the main advantages of the SMC in terms of speed and robustness without having its disadvantages while retaining its simplicity of implementation. Since the chattering appeared only in permanent regimes, the SMC will be activated during start-up and transient regimes. As the system approaches its equilibrium point, the PIAW will take over and control the steady state. A new efficient linear supervisor is responsible for determining the activation percentage of each regulator. It receives the information on speed static-error to provide an appropriate decision for high-performance control. The asymptotic stability of the hybrid controller is ensured using the second stability theorem of Lyapunov. It should be noted that the supervisor used in this technique has a simple structure that does not require a large computational load compared to the fuzzy supervisors used in [14, 18], which does not significantly complicate the overall algorithm. The new hybrid regulator will be integrated in the vector control technique for the speed regulation, while others PIAWs will control stator current. It is worth noting that this switching control will be performed without taking into account the information on rotor flux and load torque in order to simplify the algorithm and reduce its sensitivity to parametric variations.

In this context, the study will be started by a general description of the controlled system. In Section 3, the synthesis of the hybrid control is introduced and applied to the IM. After a stability analysis, simulation tests are carried out to justify the control efficiency and its robustness against parametric variations, where the results are presented in Section 4. After the experimental validation, a brief comparative study based on several performance indices was performed to show the improvement of the proposed strategy. The results are then thoroughly analyzed and discussed to justify the high efficiency of the hybrid control. In Section 5, the most important contributions are grouped in the conclusion.

\section{Mathematical model of the controlled system}

The vector control (IFOC) principal consists in orienting the rotor flux vector towards the direct plane axis in a synchronously " $d-q$ " reference frame. The rotor flux will be simplified into a single component. Therefore, the electromagnetic torque equation will be similar to the DC machine [7]. If the rotor flux module is set to its nominal value, the mechanical speed can be controlled only by the quadrature component of the stator current. This can remove some non-linearity in the mathematical model, which can be represented by Eqs. (1) to (6) $[1,4,5]$ :

$$
v_{d s}=\left(r_{s}+\delta \times L_{s} \times p\right) \times i_{d s}-\omega_{s} \times \delta \times L_{s} \times i_{q s}+\frac{M \times \psi_{r}}{L_{r}} \times p,
$$


$v_{q s}=\left(r_{s}+\delta \times L_{s} \times p\right) \times i_{q s}+\omega_{s} \times \delta \times L_{s} \times i_{d s}+\omega_{s} \times \frac{M \times \psi_{r}}{L_{r}}$,

$\psi_{r}=\frac{M}{\tau_{r} \times p+1} \times i_{d s}$

$\omega_{s}=n p \times \Omega_{m}+\frac{M}{\tau_{r} \times \psi_{r}} \times i_{q s}$,

$p \times \Omega_{m}=\frac{1}{J} \times T e-\frac{1}{J} \times T_{L}-\frac{B}{J} \times \Omega_{m}$,

$T e=\frac{3 \times M}{2 \times L_{r}} \times n p \times \psi_{r} \times i_{q s}$.

Where " $v_{d s}$ " and " $v_{q s}$ " are $d$-axis and $q$-axis stator voltages, " $i_{d s}$ " and " $i_{q s}$ " represent $d$-axis and $q$-axis stator currents, " $\psi_{r}=\psi_{d r} "$ is the instantaneous rotor flux value. $" r_{s}$ " and $" r_{r} "$ are stator and rotor resistance respectively, " $L_{s}$ ", " $L_{r}$ " and " $M$ " indicate stator, rotor and mutual inductance respectively. " $\omega_{s} ", " \omega_{s l}$ " and $" \omega_{m}$ " are respectively the synchronous angular velocity, slip angular velocity, and rotor angular velocity. " $\Omega_{m}$ " represents the instantaneous mechanical speed. "Te" and " $T_{L}$ " indicate respectively the electromagnetic torque and the load torque. " $J ", " B "$ and " $n p "$ are respectively total inertia, friction coefficient, and pole-pairs number. " $\tau_{r}=L_{r} / r_{r}$ " is the rotor time constant, where " $\delta=1-M^{2} /\left(L_{s} \times L_{r}\right)$ " is the leakage coefficient and " $p$ " refers to the Laplace operator.

\section{Nonlinear hybrid control of Induction Motor}

Fig. 1 illustrates the block diagram of the adopted IFOC structure. The motor control will be achieved using the indirect vector technique. The rotor flux is imposed directly without regulation in order to generate the reference current $" i_{d s}^{*}$ ". This component will be controlled by a PIAW regulator, which ensures the orientation of the rotor flux vector. The second regulation stage will be carried out through a series control chain. The hybrid regulator is responsible for controlling the mechanical speed; it can deliver a reference torque capable of providing a desired dynamic response. This information will be used to calculate the control frequency using Eq. (4). Then, the current " $i_{q s}^{*}$ " will be integrated into the PIAW current regulator to generate the control voltages " $v_{d s}^{*}$ " and " $v_{q s}^{*}$.

\subsection{Design of the hybrid speed controller}

\subsubsection{Steady state control}

The choice of PIAW to control the steady state is done thanks to the quality of its signals transmitted. It is considered an enhanced version of classical PI controllers. This last type can cause bad behavior (because of the Windup phenomenon) in the presence of saturation blocks, which is essential for systems protection. The windup can be caused by the integral action that continues the integration even if the control signal exceeds the maximum values [19]. This can cause a large overshoot with a high starting current. Fig. 2 illustrates the adopted PIAW design. Its structure has a return condition controlled by " $k_{r}$,

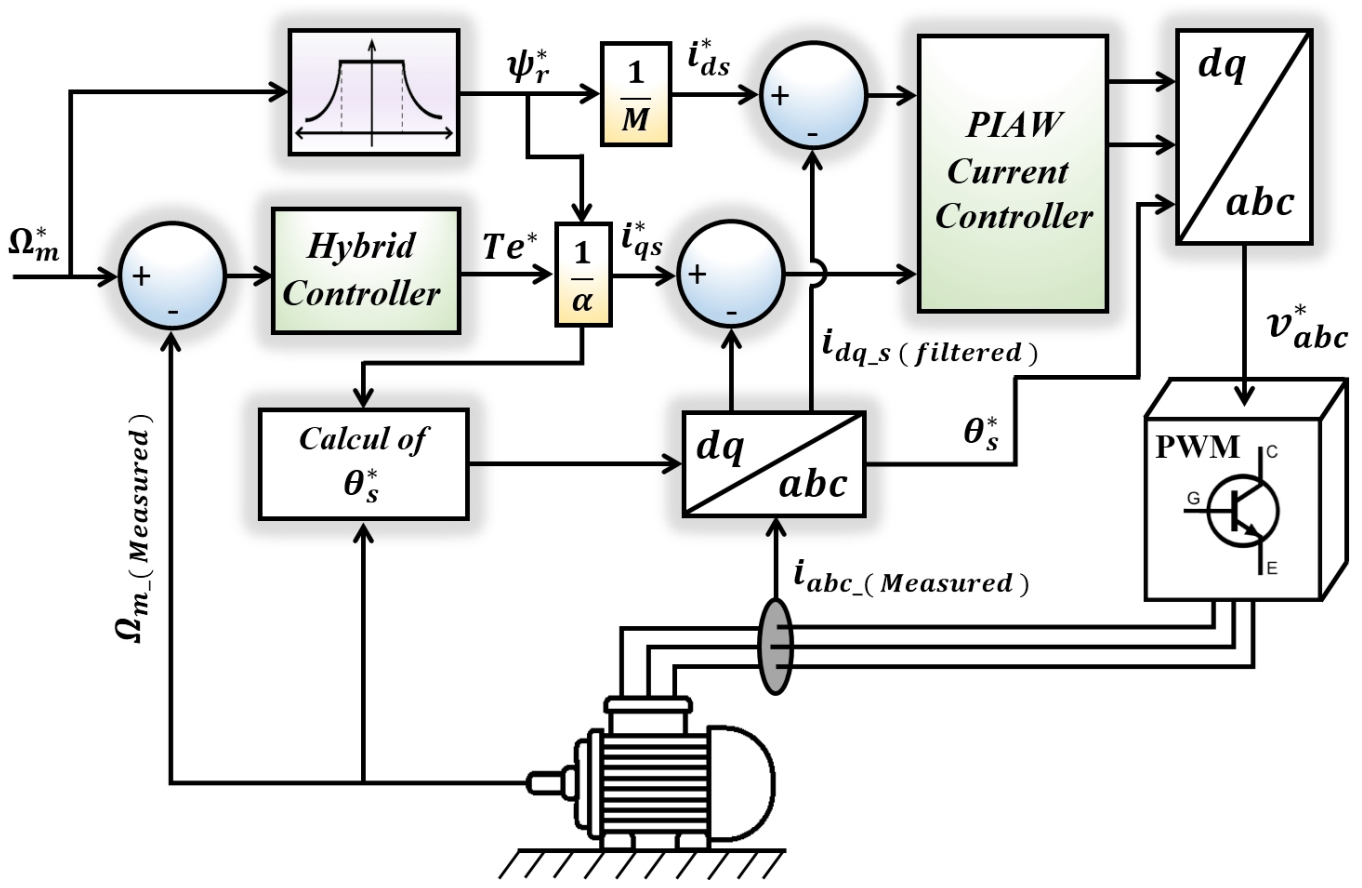

Fig. 1 Block diagram of adopted IFOC structure 


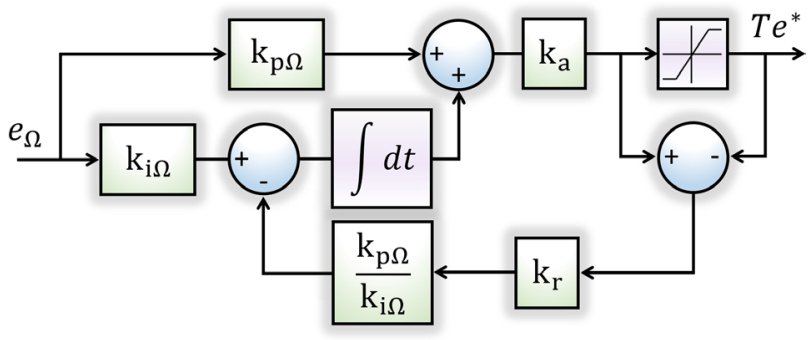

Fig. 2 Design of the PIAW used in speed control

making it possible to weaken the integral action in the case where the global control reaches its limitation values; consequently, the overtaking will be eliminated. In addition, it has an amplifier gain " $k_{a}$ " that allows adjusting both gains at the same time to facilitate the gains adjustment in real-time applications.

The conventional method can be used to determine the regulator gains. Assuming that the load torque is an unmeasurable external disturbance. The transfer function that relates rotor speed with electromagnetic torque can be expressed using Eq. (5). The denominator of the closed-loop transfer function is written as follows:

$H_{\Omega}(p)=p^{2}+\frac{B+k_{p \Omega}}{J} \times p+\frac{k_{i \Omega}}{J}$.

Using the pole placement method, the regulator gains are calculated by Eq. (8) and Eq. (9):

$k_{p \Omega}=\left(2 \times J \times \xi_{\Omega} \times \omega_{0 \Omega}\right)-B$,

$k_{i \Omega}=J \times \omega_{0 \Omega}^{2}$,

where " $\xi_{\Omega}$ " and " $\omega_{0 \Omega}$ " are the damping factor and the natural frequency of the desired system dynamic.

\subsubsection{Transient regimes control}

The operating principle of the SMC is based on the development of a control law which includes two terms. The first one ensures the attractiveness of the surface towards its equilibrium point, and the second one maintains the asymptotic stability of the system in the steady state [9]. Using the equation of Slotine and Coetsee [20], the sliding surface " $S$ " can be expressed by Eq. (10):

$S=e_{\Omega}=\Omega_{m}^{*}-\Omega_{m}$,

where $" \Omega_{m}$ " is the instantaneous value of mechanical speed, while " $\Omega_{m}^{*}$ " represents its reference. We can assume that the load torque is an unknown and limited nonlinear quantity, where its limit is the maximum load torque supported by the motor. The latter can be neglected in the presence of the integral action of global control [4]. If we use Eq. (5) and the time derivative of Eq. (10), we get:

$\frac{d S}{d t}=\frac{d \Omega_{m}^{*}}{d t}-\frac{1}{J} \times T e+\frac{B}{J} \times \Omega_{m}$.

The global control of SMC is expressed by Eq. (12). It is composed of the discontinuous term shown in Eq. (13) and the equivalent control which is determined according to the sliding mode invariance condition " $S=d S / d t \simeq 0$ " [10]:

$$
\begin{aligned}
& T_{\mathrm{SMC}}^{*}=T_{d}+T_{e q}, \quad(12) \\
& T_{d}=k_{\mathrm{SMC}} \times \operatorname{Sign}(S), \\
& T_{e q}=J \times \frac{d \Omega_{m}^{*}}{d t}+B \times \Omega_{m} .
\end{aligned}
$$

" $k_{\mathrm{SMC}} "$ is the attraction gain of the Sliding Mode Controller which is purely positive and judiciously chosen in order to make the control capable of eliminating external disturbances, and on the other hand, it must respect the maximum torque supported by IM.

\section{Chattering phenomenon reduction}

The chattering phenomenon consists in switching from a maximum value to a minimum with very high frequency. The conventional SMC can offer a good performance only for large gains. However, this condition can complicate the elimination of the chattering in control signals. One proposed solution consists in the replacement of the discontinuous function "Sign" by the continuous one "Smooth" " $\mathcal{F}_{s}(S)$ ". It can be expressed by Eq. (15), where " $\sigma$ " is a constant that defines the degree of oscillation weakening, which must be chosen to be very small. Fig. 3 illustrates the control transition using "Smooth" function:

$\operatorname{Smooth}(S)=\mathcal{F}_{s}(S)=\frac{S}{|S|+\sigma}$.

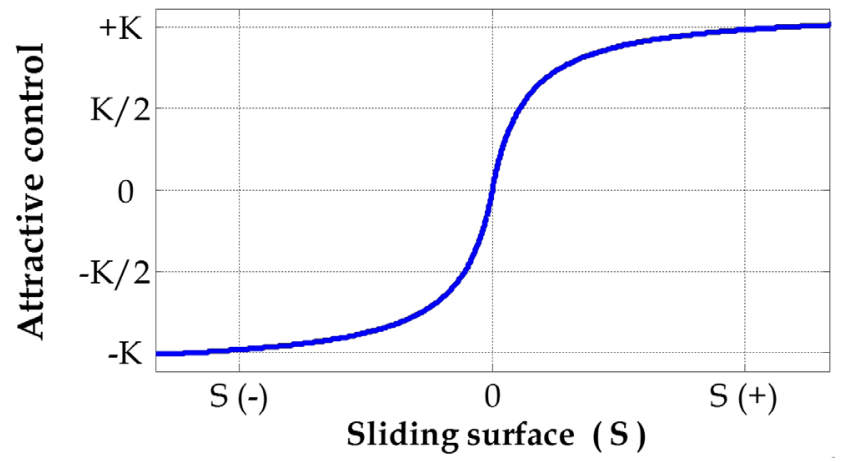

Fig. 3 Commutation of the discontinuous command based on "Smooth" function 


\subsubsection{Switching control implementation}

All controllers will be operated at the same time with a different activation percentage. The supervisor that activates each regulator in its operation zone can deliver information ranges from " 0 " to " 1 " called the decision " $d$ ". This latter is an element which strictly affects the control law generated by the hybrid regulator and expressed by Eq. (16):

$T e^{*}=d \times T_{\mathrm{SMC}}^{*}+(1-d) \times T_{\mathrm{PIAW}}^{*}$.

Note that " $T e^{*} "$ is the final speed control, while " $T_{\text {SMC }}^{*}$ and " $T_{\mathrm{PIAW}}^{*}$ " are partial controls that are delivered by SMC and PIAW respectively. The gradual switching can maintain the overall stability as it avoids the instantaneous passage from one regulator to another. Fig. 4 gives a graphical representation of the supervisor output, which is developed using the algorithm presented in Algorithm 1. Its simplicity allows reducing the computing load to ensure optimal switching between the two controllers, where " $e_{\Omega}$ " is the speed error, " $E_{\max }$ " and " $E_{\text {min }}$ " are its maximum and minimum values respectively. The supervisor's configuration must be selected in a way to make the control sensitive to speed drops to ensure fast speed error elimination. While the supervisor remains at the value " 0 " for each

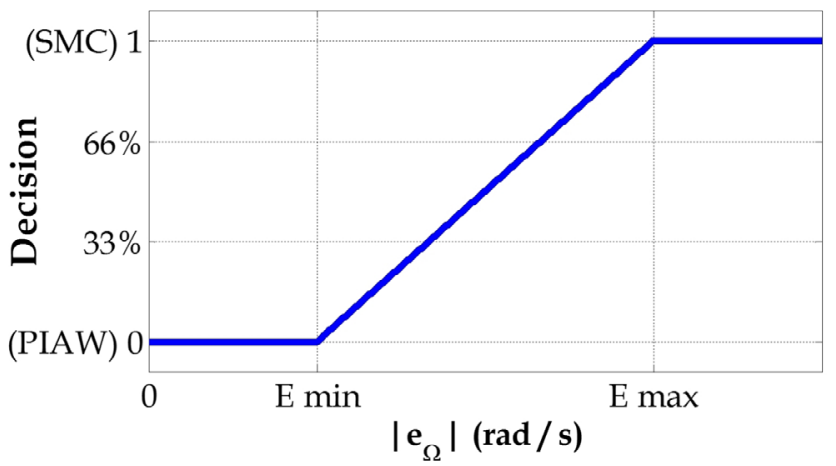

Fig. 4 Supervisor decision according to speed static-error

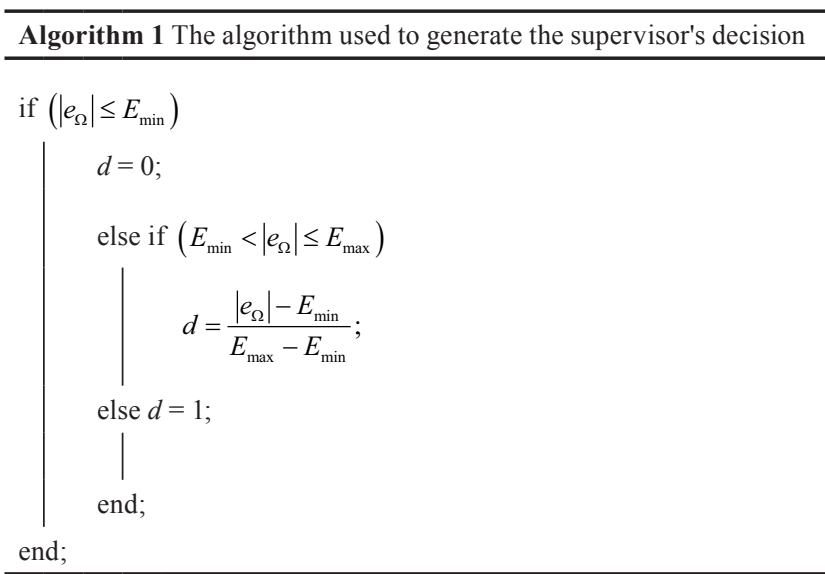

speed variation below " $E_{\min }$ " in order to improve the quality of the control signal (by using the PIAW) and eliminate the Chattering phenomenon.

\subsubsection{Stability analysis}

According to the development of each regulator, we can write:

1. The PIAW regulator is stable since the transfer-function poles have the real parts strictly negative. Its delivered control has the following form:

$$
T_{\mathrm{PIAW}}^{*}=k_{a} \times\left(k_{p \Omega} \times S+k_{i \Omega} \int S d t\right) .
$$

2. The information transmitted by the supervisor has a bounded variation, which varies between [0 1].

In order to analyze the overall stability of the hybrid regulator, we can use the following Lyapunov function:

$$
\begin{aligned}
& \forall\{S \neq 0, \quad \mathcal{X} \neq 0\}, \quad V_{h}=\frac{1}{2} \times S^{2}+\frac{1}{2} \times \mathcal{X}^{2}, \\
& \mathcal{X}=\sqrt{L_{h}} \times \int S d t, \quad \forall L_{h}=\frac{k_{a} \times k_{i \Omega} \times(1-d)}{J} .
\end{aligned}
$$

By neglecting the variation of the supervisor's decision, the derivative of the Lyapunov function can be expressed by Eq. (19) using Eq. (11).

$$
\frac{d V_{h}}{d t}=S \times\left(\frac{d \Omega_{m}^{*}}{d t}-\frac{1}{J} \times T e^{*}+\frac{B}{J} \times \Omega_{m}+L_{h} \times \int S d t\right) .
$$

Substituting Eq. (12), Eq. (16) and Eq. (17) in Eq. (19), we obtain:

$$
\begin{aligned}
& \frac{d V_{h}}{d t}=S \times\left[\begin{array}{l}
-\frac{d \times k_{\mathrm{SMC}}}{J} \times \mathcal{F}_{s}(S)-\frac{(1-d)}{J} \times k_{a} \times k_{p \Omega} \times S \\
+(1-d)\left(\frac{d \Omega_{m}^{*}}{d t}+\frac{B}{J} \times \Omega_{m}\right)
\end{array}\right] \\
& =\left[-\frac{d \times k_{\mathrm{SMC}}}{J} \times|S|+S \times(1-d)\left(\frac{d \Omega_{m}^{*}}{d t}+\frac{B}{J} \times \Omega_{m}\right)\right] \\
& -\frac{(1-d)}{J} \times k_{a} \times k_{p \Omega} \times S^{2} .
\end{aligned}
$$

To check the asymptotic stability, it is necessary that:

$$
-\frac{d \times k_{\mathrm{SMC}}}{J} \times|S|+S \times(1-d)\left(\frac{d \Omega_{m}^{*}}{d t}+\frac{B}{J} \times \Omega_{m}\right) \leq 0 .
$$

We can conclude the following stability condition:

$$
k_{\mathrm{SMC}} \geq \operatorname{Max}\left\{\frac{(1-d)}{d} \times\left(J \times \frac{d \Omega_{m}^{*}}{d t}+B \times \Omega_{m}\right)\right\} .
$$


- For " $d=0$ ": The control will be carried out by the PI Anti-Windup regulator, so the stability of the system is ensured according to the pole-placement theorem.

- For " $d \neq 0$ ": The system will be asymptotically stable if the gain of the discontinuous control " $k_{\mathrm{SMC}}$ " is selected to be relatively large.

We can therefore conclude that, regardless of the operating zone of the machine, the hybrid controller makes the speed converge towards its reference and ensures that zero is the equilibrium point for the static error of the mechanical speed.

\subsection{Current regulation}

The measured Park currents that are intended for current regulation may be of poor quality because of the presence of the various disturbances, mainly due to current sensors, analog to digital converter, Park transformations as well as the operating principle of the inverter. For this, it is not recommended to use the same hybrid regulator in the current regulation chain because the supervisor's decision becomes imprecise, which risks likewise diverging the system response. Therefore, it is very appropriate to adopt a PIAW regulator to perform this regulation. The reference currents can be given by Eq. (23):

$$
i_{d s}^{*}=\frac{1}{M} \times \psi_{r}^{*},
$$$$
i_{q s}^{*}=\frac{2 \times L_{r}}{3 \times M \times n p} \times \frac{T e^{*}}{\psi_{r}^{*}} .
$$

Equations (1) and (2) which relate the supply voltages to stator currents are clearly nonlinear. A proposed solution has been introduced in [1] to perform control based on the transfer functions. It is known that the current dynamic is very fast compared to that of rotor flux. We can therefore neglect the latter in Eq. (1). In addition, the coupling terms in Eq. (1) and Eq. (2) can be removed to arrive at a first-order relation between voltages and currents. After calculating the gains of regulators, the coupling terms are added in the regulation chain output. This method can provide good control and maintains the global stability of the system. The controlled sub-system can be described by Eq. (24) with " $\tau_{s}=L_{s} / r_{s}$ ":

$$
G_{O L}(p)=\frac{i_{d q}}{v_{d q}}=\frac{1}{r_{s}} \times \frac{1}{\left(\delta \times \tau_{s} \times p+1\right)} .
$$

In order to improve the current regulation quality, a simple low-pass filter (with a cut-off frequency
" $f_{c}=1 / T_{c}$ ") was implemented to minimize fluctuations presented in the actual current. We arrive at the block diagram shown in Fig. 5.

The closed-loop transfer function can be simplified if we chose " $k_{p l} / k_{i i}=\delta \times \tau_{s}$ ". It can be expressed by:

$G_{C L}(p)=\frac{\frac{k_{i i}}{\left(r_{s} \times T_{c}\right)} \times\left(T_{c} \times p+1\right)}{p^{2}+\frac{1}{T_{c}} \times p+\frac{k_{i i}}{\left(r_{s} \times T_{c}\right)}}$.

The filter cut-off frequency should be chosen to be relatively large to remove only high frequencies without affecting the current regulation by introducing delays. In this condition, the zero of the transfer function has a weak influence on the system dynamics [4]. Using the pole-placement method, the regulator gains can be calculated by Eq. (26):

$k_{i i}=\frac{r_{s}}{4 \times T_{c} \times \xi^{2}}$,
$k_{p i}=\delta \times \tau_{s} \times k_{i i}$.

The anti-windup option has been introduced in this regulation by using the same regulator shown in Fig. 2, and the deleted coupling terms have been added to the control chain outputs. The final control voltages can be written as:

$$
\begin{aligned}
& v_{d s}^{*}=k_{a i} \times\left(k_{p i} \times e_{d}+k_{i i} \times \int e_{d} d t\right)-\omega_{s} \times \delta \times L_{s} \times i_{q s}^{*} \\
& v_{q s}^{*}=k_{a i} \times\left(k_{p i} \times e_{q}+k_{i i} \times \int e_{q} d t\right)+\omega_{s} \times \delta \times L_{s} \times i_{d s}^{*} \\
& +\omega_{s} \times \frac{M}{L_{r}} \times \psi_{r}^{*} .
\end{aligned}
$$

\section{Results and discussion}

In order to validate the theoretical study, a series of simulations are carried out with the aim of verifying the control performance in different operation zones and under different conditions. The hybrid control is applied to a $1 \mathrm{~kW}$ IM where its parameters are mentioned in Table 1. The static fourth-order method is used to solve different dynamic equations with a computation time of $10 \mu$ s. After carrying out robustness tests for parametric

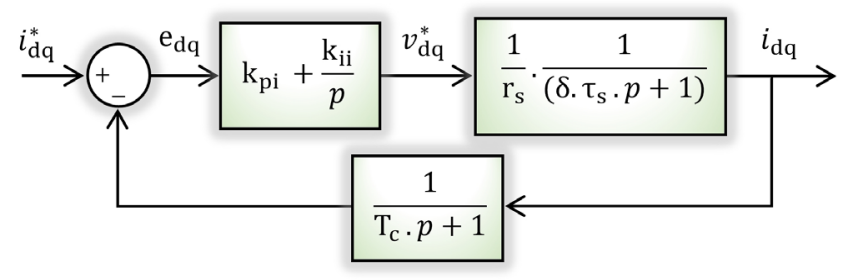

Fig. 5 Current regulation loop 
Table 1 Induction Motor parameters

\begin{tabular}{lccc}
\hline$\Omega_{n}(\mathrm{rad} / \mathrm{s})$ & $v_{n}(\mathrm{~V})$ & $i_{n}(\mathrm{~A})$ & $f_{n}(\mathrm{~Hz})$ \\
\hline 145 & $220 / 380$ & $2.5 / 4.32$ & 50 \\
\hline$r_{s}(\Omega)$ & $L_{s}(\mathrm{H})$ & $L_{r}(\mathrm{H})$ & $M(\mathrm{H})$ \\
8.79 & 0.868 & 0.072 & 0.240 \\
\hline$r_{r}(\Omega)$ & $J\left(\mathrm{~kg} \mathrm{~m}^{2}\right)$ & $B(\mathrm{~N} \mathrm{~m} \mathrm{~s} / \mathrm{rad})$ & $n p$ \\
0.65 & 0.0157 & 0.0045 & 2 \\
\hline
\end{tabular}

variations, the experimental validation is necessary to justify the possibility of achieving variable speed drive based on this technique. The same algorithm used in the simulation will be implemented in a dSPACE-RTI-1104 card. The hybrid regulator adopted in the speed control is illustrated in Fig. 6. The gains values and the experimental configuration of each part of the global control are given in Table 2.

The method used to define the gains of the PIAW regulators (either for speed or for current regulation) is based on the pole placement strategy by taking a damping factor $\xi=1 / \sqrt{2}$ that corresponds to an overshoot of less than $5 \%$. The natural frequency " $\omega_{0}$ " of the desired canonical forms must respect the condition that the current regulation dynamic must be 10 times faster than that of the speed regulation dynamic. On the other hand, the SMC gain was selected in a way that allows developing a reference torque capable of eliminating the maximum values of external disturbances, which can be expressed by the maximum value of the applied loads supported by the motor. We note also that, the supervisor configuration is: $E_{\min }=0.9$ and $E_{\max }=4.0$. This means that for a speed difference less than $0.9 \mathrm{rad} / \mathrm{s}$, we can consider that the mechanical speed remains around the equilibrium point. Furthermore, it is practically justified that an uncertainty lower than
$0.9 \mathrm{rad} / \mathrm{s}$ can be considered as a natural static error due to several factors not included in the analytical development, especially because of the imperfection of the harmonically-rich voltages delivered by the inverter. Moreover, if the error is greater than $0.9 \mathrm{rad} / \mathrm{s}, \mathrm{SMC}$ will be intervened by a different percentage. If the error is greater than $4 \mathrm{rad} / \mathrm{s}$, the SMC will operate alone as in the case of the start-up.

The experimental configuration shown in Fig. 7 consists of a $1 \mathrm{~kW}$ three-phase IM with a star " $Y$ " connection. It is mechanically coupled with a powder brake to ensure the application of load torques. The motor is powered by a PWM inverter with a DC bus of $550 \mathrm{~V}$, and a switching frequency of $3 \mathrm{kHz}$. The inverter control circuit receives signals via the dSPACE interface. The stator currents and the mechanical speed are measured through Hall-effect sensors and an incremental encoder. The control algorithm uses this information to generate control voltages appropriate to the desired dynamics. The ControlDesksoftware can control the motor and allow the observation of its dynamics in real-time. The dynamic equations of the algorithm are solved by using the first-order differential equations (Euler) method with a time step size of $175 \mu \mathrm{s}$.

\subsection{Experimental comparative study}

In order to evaluate the performance of the proposed hybrid technique, a comparative study with the PIAW was carried out. It assessed the following criteria: simplicity, overshooting percentage, and speed drops due to applied load torques. The study also used the following performance indices: Integral Absolute Error (IAE), Integral Square Error (ISE), and Integral Time Absolute Error (ITAE) as expressed in Eq. (28). The results of the comparative study are presented in Table 3.

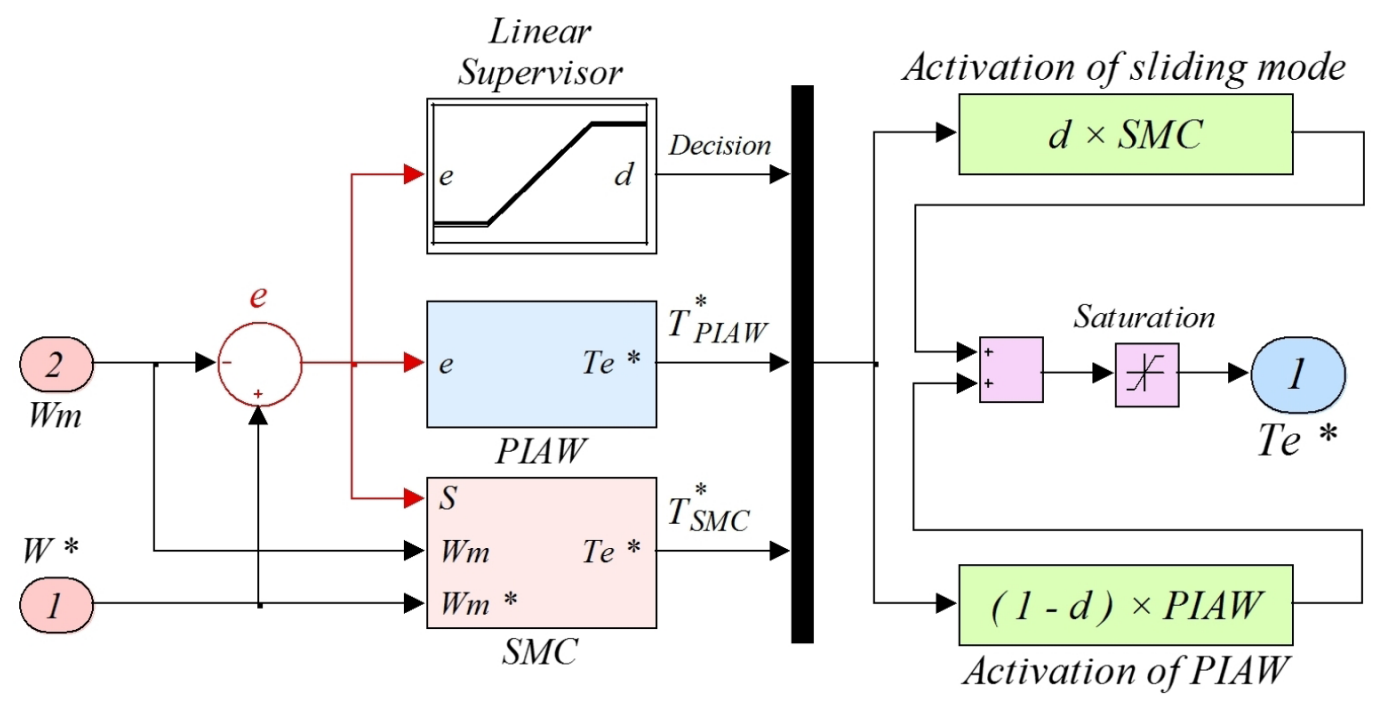

Fig. 6 Hybrid regulator design adopted in speed control 
Table 2 Experimental configuration of the control algorithm

\begin{tabular}{|c|c|c|c|}
\hline \multicolumn{2}{|c|}{ Hybrid speed controller } & \multirow{2}{*}{ Supervisor } & \multirow{2}{*}{ PIAW current regulator } \\
\hline SMC & PIAW & & \\
\hline \multirow{4}{*}{$k_{\mathrm{SMC}}=5.0$} & $k_{p \Omega}=0.5$ & \multirow{2}{*}{$E_{\max }=4.0$} & $k_{p i}=4.0$ \\
\hline & $k_{i \Omega}=3.0$ & & $k_{i i}=450.0$ \\
\hline & $k_{a}=2.0$ & \multirow{2}{*}{$E_{\min }=0.9$} & $k_{a i}=1.0$ \\
\hline & $k_{r}=2.0$ & & $k_{r i}=1.0$ \\
\hline
\end{tabular}

$\mathrm{IAE}=\int_{0}^{T_{\text {Final }}}\left|e_{\Omega}\right| d t$

$\mathrm{ISE}=\int_{0}^{T_{\text {Final }}} e_{\Omega}^{2} d t$

$\mathrm{IATE}=\int_{0}^{T_{\text {Final }}} t \times\left|e_{\Omega}\right| d t$

\subsection{Discussion}

The new control algorithm was tested under conditions designed to verify the motor's performances at the presence of different external and internal disturbances. Fig. 8 and Fig. 9 illustrate the control performance of the IM under nominal conditions. We can note a good speed tracking with a high disturbance rejection capacity. Load torque applications cause a small speed drop, which is quickly eliminated as it appeared at instant $0.5 \mathrm{~s}$ and $1.5 \mathrm{~s}$. The good speed regulation appears on the static error figure, which shows that the difference between the mechanical speed and its reference remains very small in all different operating zones. It is also remarkable that the electromagnetic torque developed by the motor (which follows its reference perfectly) does not exhibit strong fluctuations. This is due to the high quality of the signal delivered by the hybrid regulator free of chattering. Since this reference signal consists of two partial commands, the SMC is activated during transient regimes (e.g. instants $0.1 \mathrm{~s}, 1.5 \mathrm{~s}$, and $2.2 \mathrm{~s}$ etc.) and the PIAW drives the steady state (e.g. instants $0.6 \mathrm{~s}, 1.3 \mathrm{~s}$, and $2.5 \mathrm{~s}$ etc...). This justifies the fast dynamic speed response without overshooting and the total absence of the chattering phenomenon in the control signals. These performances are obtained with the help of the new supervisor that was able to drive the control during all operation zones, by generating a stable decision that can ensure good switching from one regulator to another. Note that this supervisor requires less computing time compared to that used in [13-14, 18]. It can detect the presence of external disturbances and activates the SMC

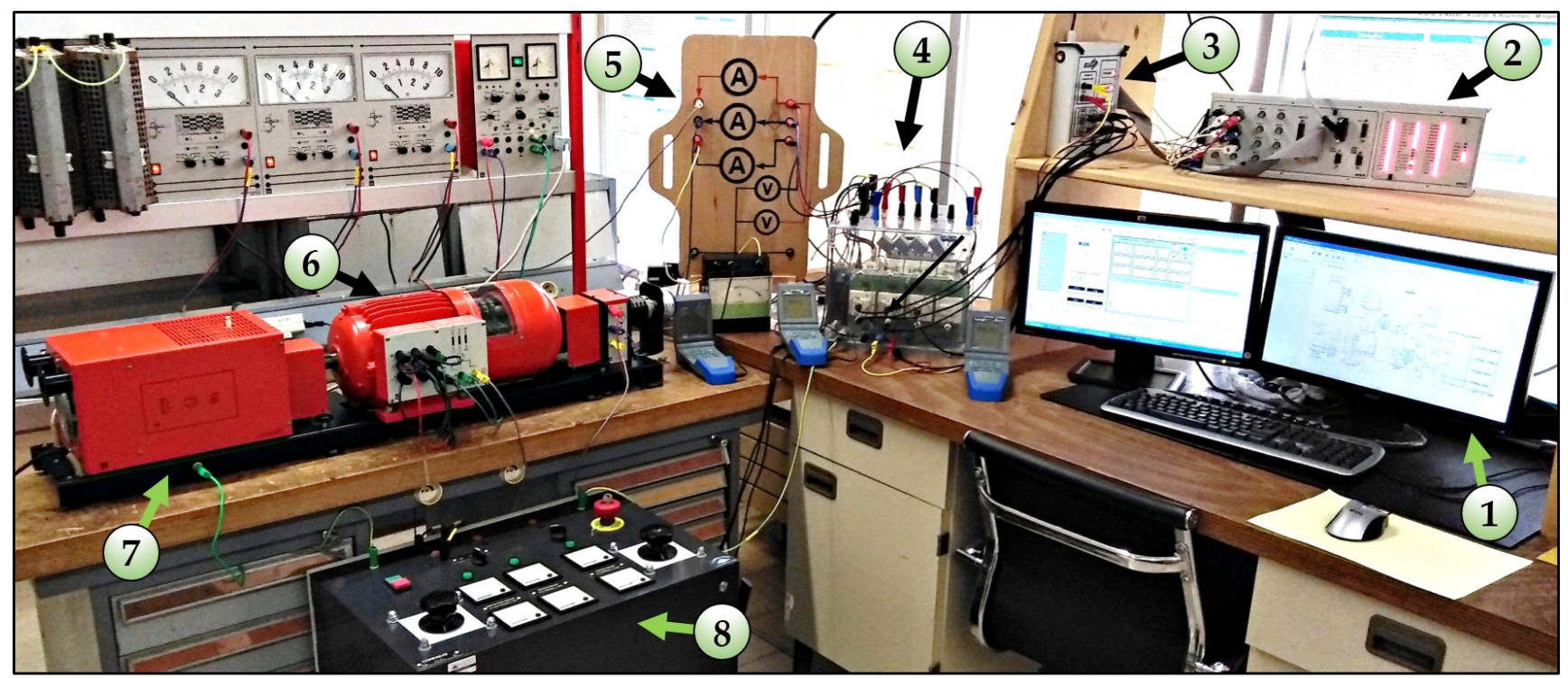
(1) - Control unit
(2) - dSPACE-RTI-1104
(3) - Control circuit of IGBT
(4) - PWM Inverter
(5) - Hall-effect sensors
(6) - Three-phase IM
(7) - Powder brake
(8) - Auto-transformer

Fig. 7 Experimental setup of LDEE-USTO laboratory (Group of Control)

Table 3 Results of the comparative study

\begin{tabular}{lcccccc}
\hline & Structure & Overtaking $(\%)$ & Maximum speed drop $(\mathrm{rad} / \mathrm{s})$ & IAE $(\mathrm{rad})$ & ISE $\left(\mathrm{rad}^{2} / \mathrm{s}\right)$ & IATE $(\mathrm{rad}$ ) \\
\hline PIAW & Simple & 4.2 & 3.5 & 11.29 & 30.7 & 178.95 \\
Hybrid control & complicated & 0 & 2.2 & 10.65 & 39.81 \\
\hline
\end{tabular}



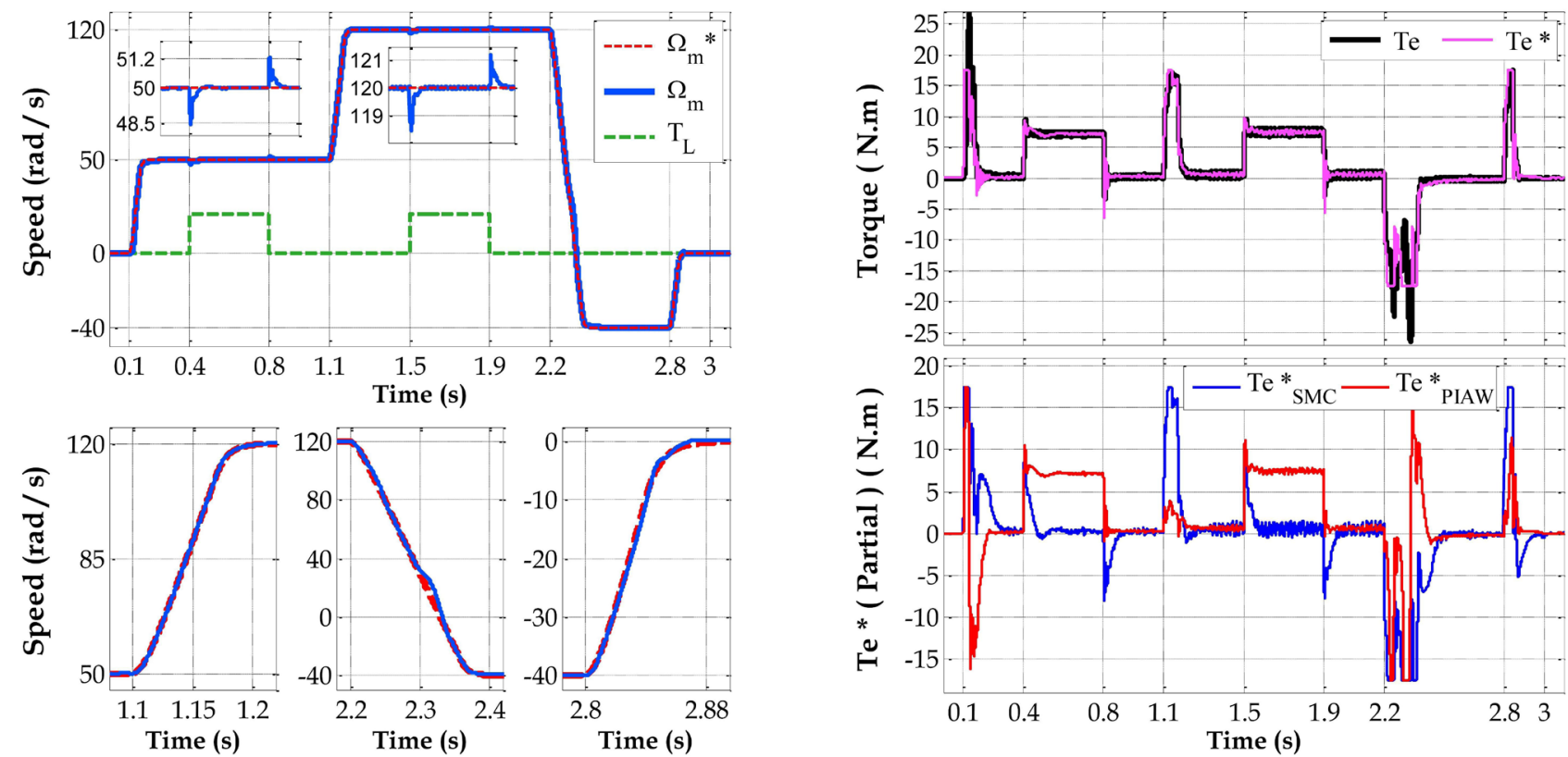

Fig. 8 Simulation results showing the control performance for nominal conditions (Part 1)
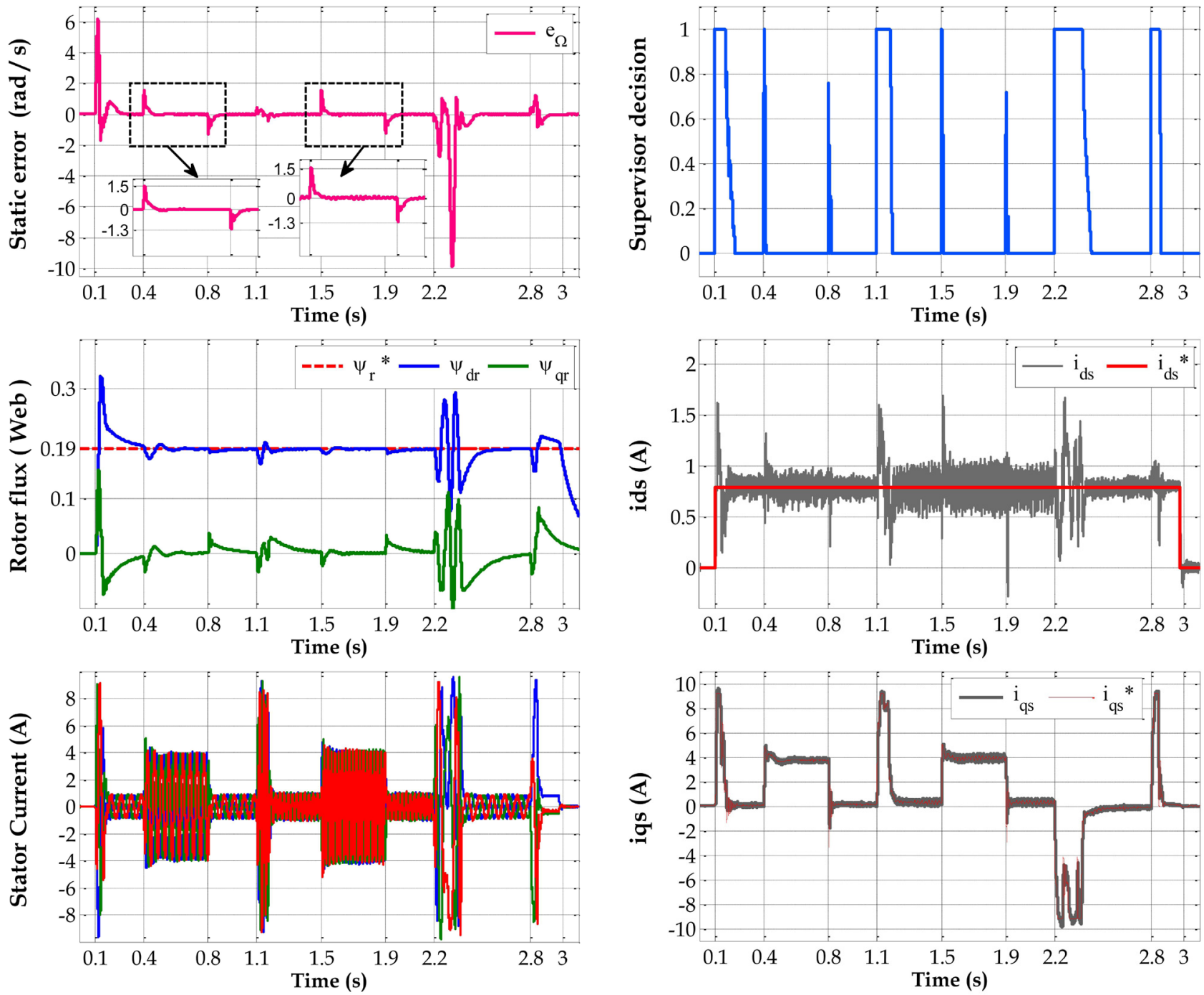

Fig. 9 Simulation results showing the control performance for nominal conditions (Part 2) 
to interfere with variable percentages linked to the speed drops. This ensures the control's robustness against applied load torques. Besides, the realization of the vector control principle is key for obtaining this performance. This is apparent in the rotor flux which is composed of a zero component and another one maintained on the imposed flux value. This directly reflects the control quality of the stator currents " $i_{d s}$ " and " $i_{q s}$ " that converge quickly with bounded values towards their references.
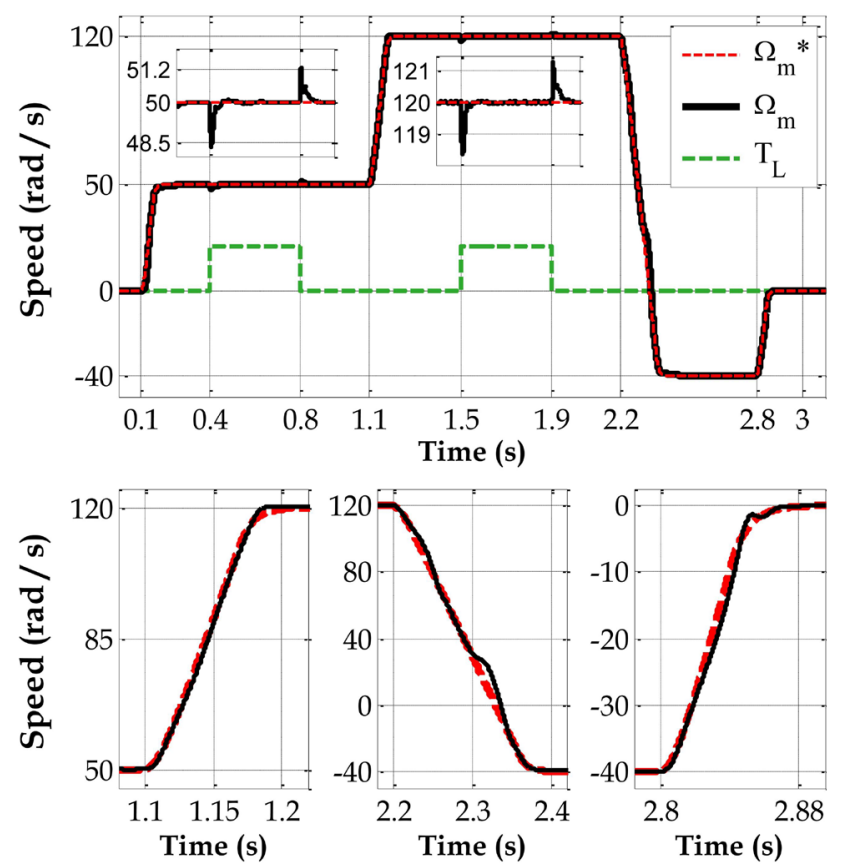

Fig. 10, Fig. 11 and Fig. 12 show the control performance in the case of parametric variations with respect to stator resistances, rotor resistances and the moment of inertia. The modification includes an increase in each parameter to reach up to $200 \%$ of its nominal value. The results show remarkable performance in terms of speed, stability, good current control and robustness against external loads applied. There is a slight expected deterioration of performance in the case of total inertia variation. The dynamic
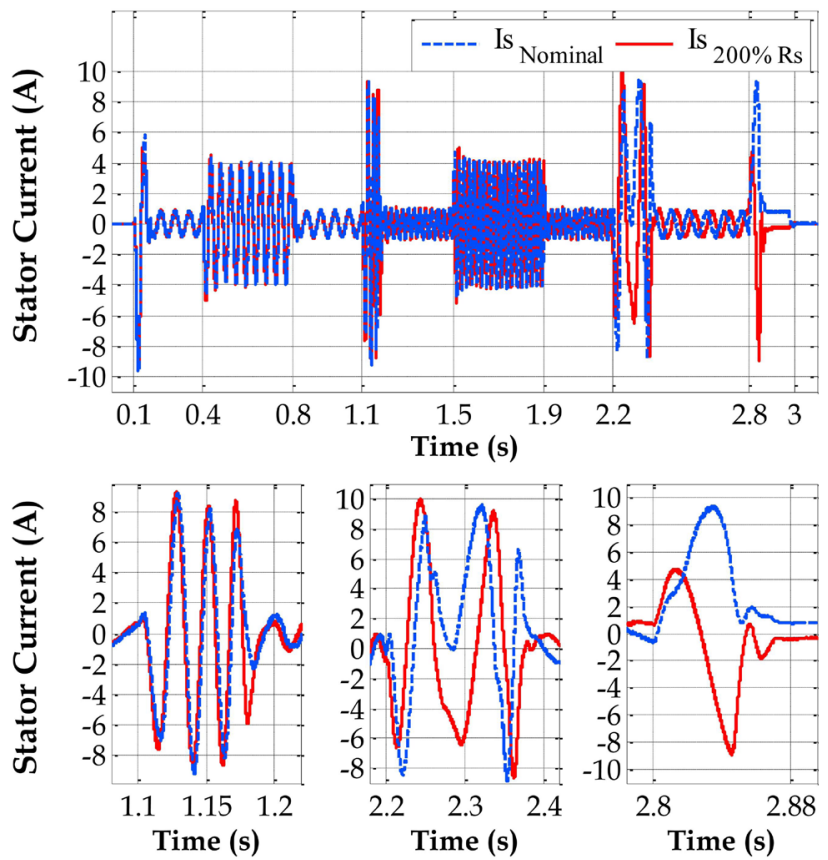

Fig. 10 Effect of stator resistance variation on speed and current regulation
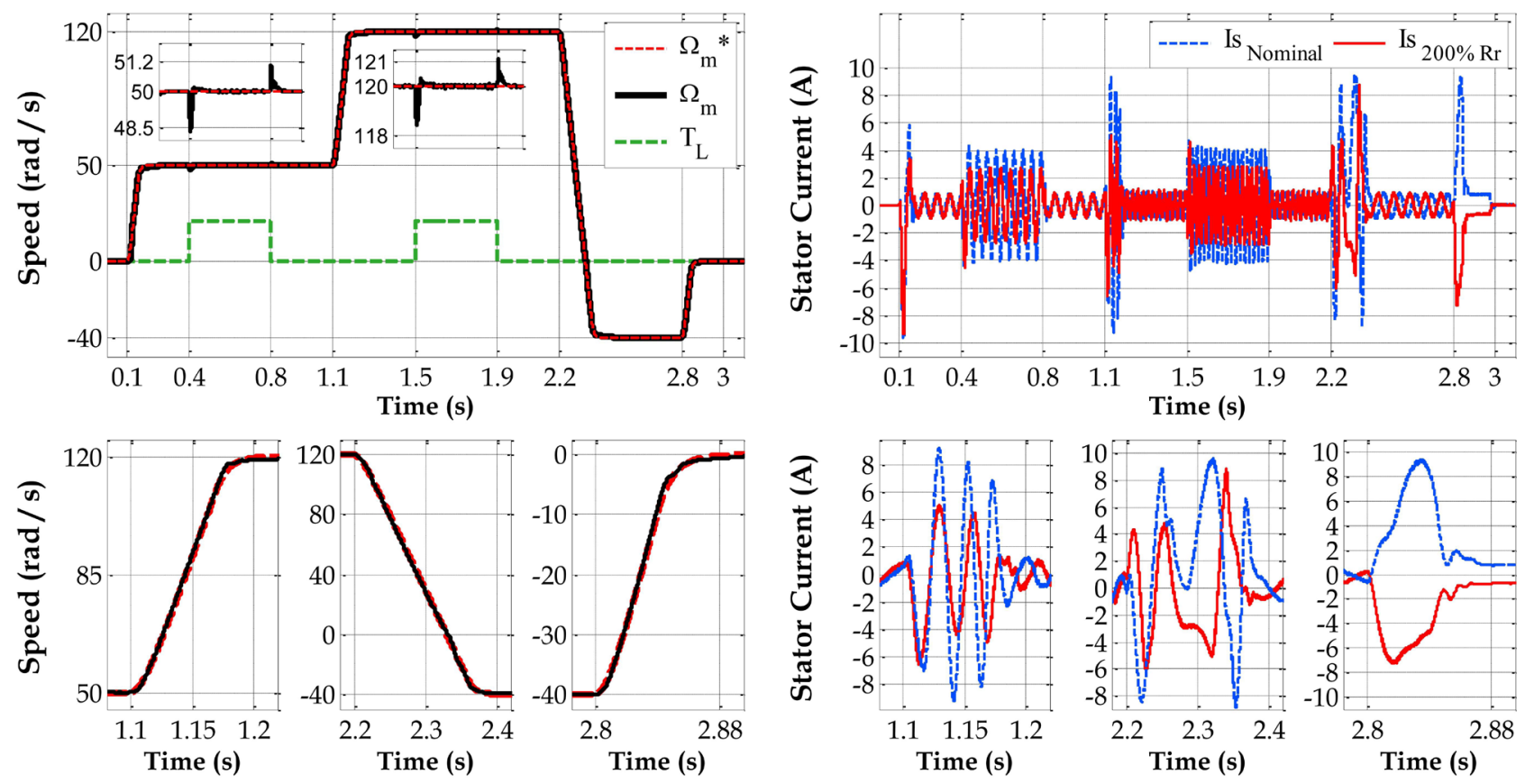

Fig. 11 Effect of rotor resistance variation on speed and current regulation 

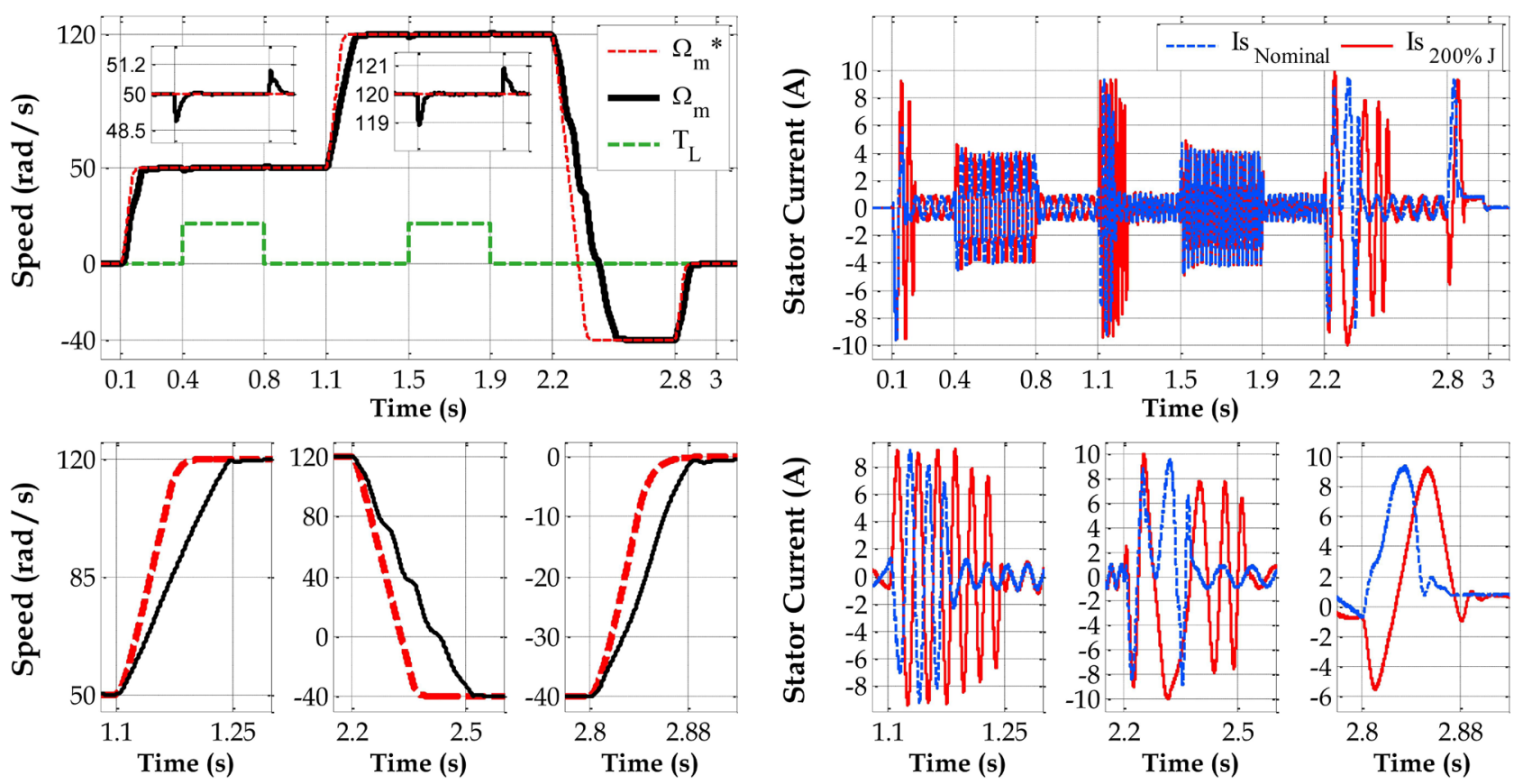

Fig. 12 Effect of total inertia variation on speed and current regulation

response is relatively slow compared to the nominal case as seen at moments $0.1 \mathrm{~s}, 2.2 \mathrm{~s}$ and $2.8 \mathrm{~s}$ in Fig. 12. There is also a small increase in stator current during transient regimes. This degradation is quite acceptable since the speed converges towards its reference with good stability, and the load torque rejection is carried out perfectly. Besides, the increase of stator current always remains within the margin supported by the motor and the inverter.

The experimental results confirm the performance obtained by simulation. The control offers a fast dynamic speed tracking, with good applied torques rejection, where the speed drop is less than $2 \%$ at high speed as it appeared in Fig. 13. Moreover, traceability in transitional regimes is extremely impressive especially in terms of stability. This property is particularly apparent in the speed gap figure. Based on Fig. 13 and Fig. 14, it can be said that the theory of the hybrid controller has been successfully implemented with high efficiency. The control signal generated by the hybrid regulator presents a stable and bounded response completely free of the chattering phenomenon, which is in line with the theoretical results. The permanent regime appears clearly to be controlled by PIAW, where the SMC intervened during transitional regimes. The proposed supervisor was able to steer the hybrid regulator and optimize its performance in real-time with no problems during all operating zones without affecting the dynamics of the stator current regulation chain and the one of rotor flux. The two components " $i_{d s}$ " and $" i_{q s}$ " followed these references perfectly with a bounded response, ensuring optimal control of the real three-phase current. The rotor flux was maintained at its reference throughout the trajectory confirming the achievement of the vector control principle since the electromagnetic torque and the rotor flux were controlled separately.

Table 3 compares the performances of the hybrid control to the one based only on PIAW. Our approach shows a significant improvement in the total elimination of overshoot and the disturbance rejection. The latter was optimized by $37 \%$ compared to the performance given by PIAW. If we look at the IAE index, we can see that the regulation error generated by the hybrid regulator was improved, as well as the stability and the conservation of performance over time (as it appears in the IATE index). Furthermore, it is expected to have a higher ISE than that of PIAW, since the latter is based on an integral action making it possible to accelerate the start-up. However, this action can cause unwanted overshoot and overcurrent in transitional regimes. On the other hand, the control improvement applied in this work can be more illustrated by comparing the present hybrid controller with other techniques based on the same principle. The authors of [13] integrated the fuzzy logic into the basic sliding mode structure in order to minimize chattering. This regulator was used in transient regimes, while a nonlinear controller was adopted for the steady state through 

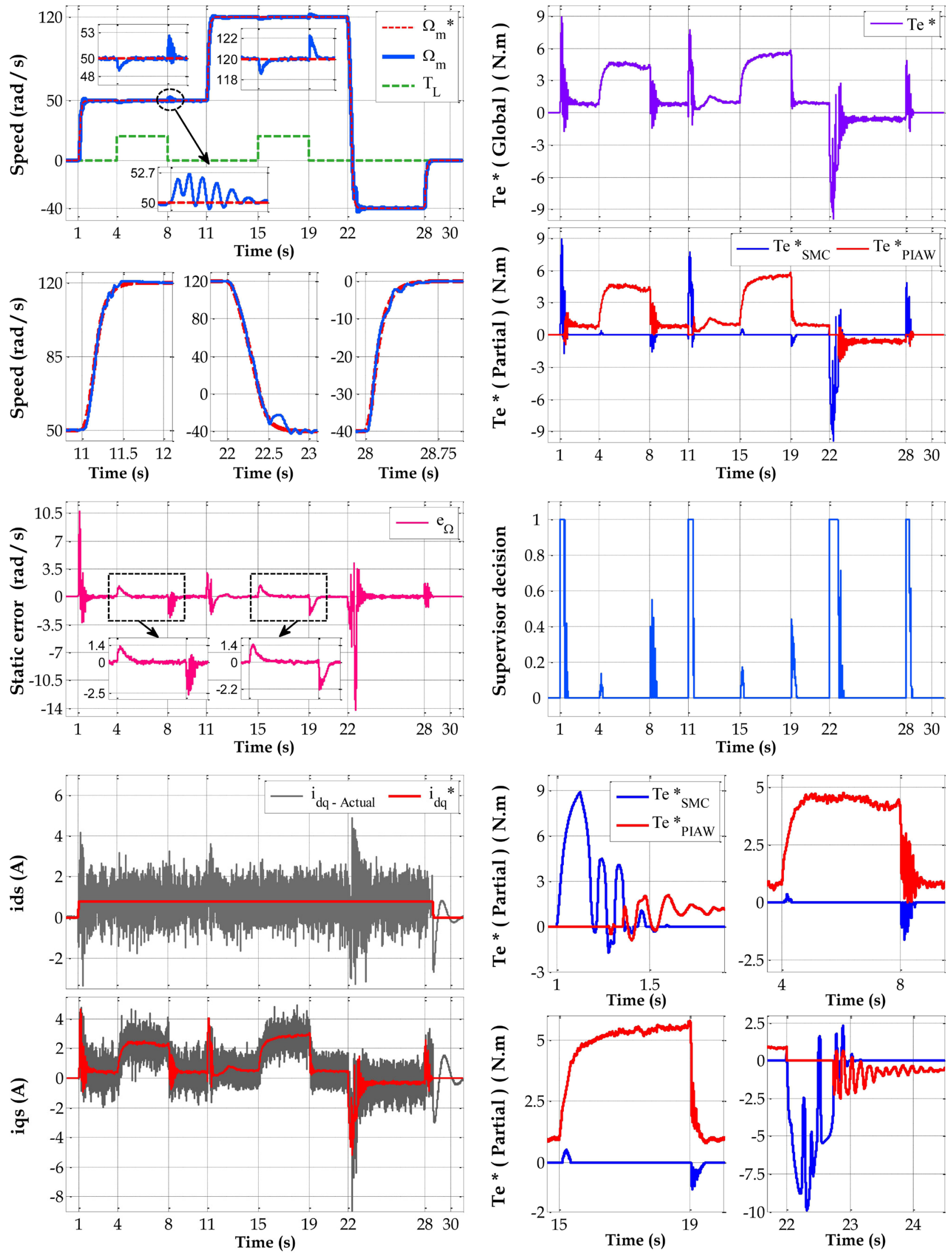

Fig. 13 Experimental results of IM performance for the proposed hybrid control (Part 1) 

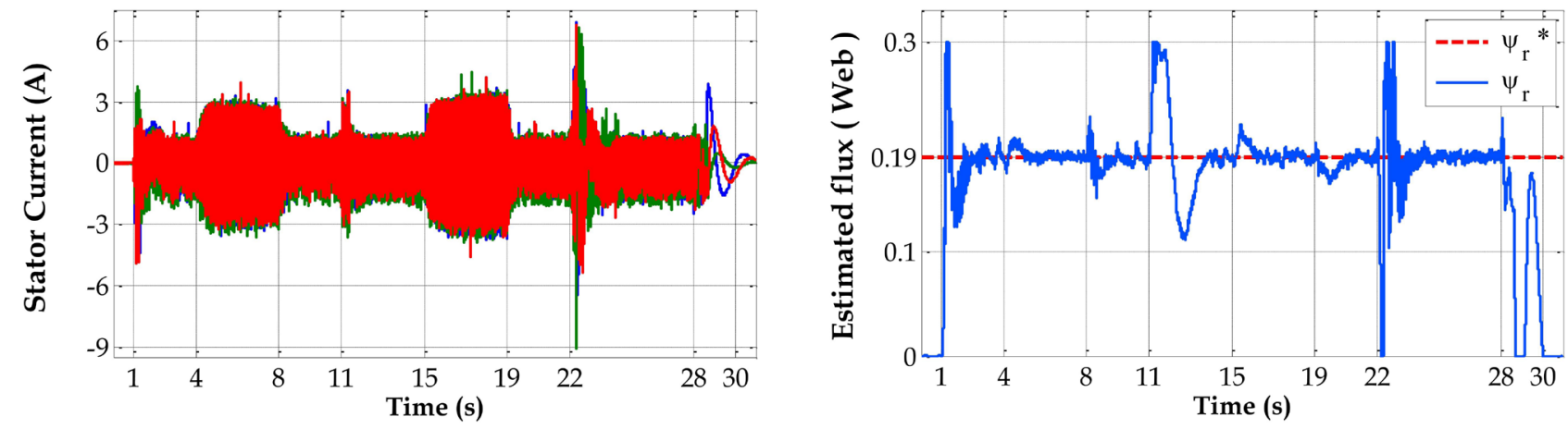

Fig. 14 Experimental results of IM performance for the proposed hybrid control (Part 2)

a two-dimensional supervisor requiring a derivative to determine the correct supervisor decision. The obtained results were highly satisfactory and quite similar to those obtained using our method. However, the cost of the algorithm was high compared to the one developed in this paper, and the overall stability of their hybrid regulator was not verified. This may introduce difficulty in a practical implementation. The work developed in this paper is intended for real applications. Its contribution is characterized by optimizing the control performance with maintaining the same degree of complexity. The simplicity of developing each control part and using the Lyapunov stability to synthesize the speed control law has made it possible to implement this technique in real-time in an easy way, thus ensuring high-performance control of all states of the three-phase Induction Motor.

\section{Conclusion}

In this paper, a new synthesis of nonlinear hybrid techniques is applied for the control of a three-phase Induction Motor speed. The proposed method combines the sliding mode with the PI Anti-Windup. The latter offers high-quality of control signals while still guaranteeing good speed and current regulation. To have better performance, a new simple supervisor is integrated in the main speed controller. It can generate an appropriate decision for the desired dynamic according to the speed's static error. This information is used to determine the activation percentage of each controller. The sliding mode is activated during start-up and transient regimes, while the PIAW controls the steady state. The overall controller stability was analyzed using the Lyapunov theorem. This method was verified by simulation and then by experimental validation using a dSPACE-DS-1104 card. The results show high-performance speed and current control, with remarkable robustness against external and structural disturbances. It should also be noted that the chattering phenomenon disappeared completely from the control signals; which means that this technique can reap the benefits of Sliding Mode Control without having these disadvantages.

As part of potential further research, we aspire to replacing the classical sliding mode with its integral version in order to enhance dynamic response during transient regimes which will improve the control. Besides the supervisor's decision can be made more efficient if we integrate the information on the speed variation. This would take the properties of a fuzzy supervisor without increasing the computational time.

\section{Nomenclature}

IM Induction motor.

SMC Sliding Mode Controller.

PIAW Proportional Integral regulator with Anti-windup.

$d, q \quad$ Indices of the synchronous reference frame.

$s, r, *$ Indices relating to stator, rotor, and reference values.

$v, i, \psi \quad$ Instantaneous values of voltage, current and flux.

$r, L, M$ Resistance, inductance and magnetizing inductance.

$J, B \quad$ Total inertia, friction coefficient.

np Pole-pair number.

$T e, T_{L}$ Electromagnetic torque and load torque.

$\omega_{s}, \omega_{s l}$ Stator and slip pulsation.

$\Omega_{m}, \omega_{m}$ Rotor speed and mechanical pulsation respectively.

p Laplace operator. 


\section{References}

[1] Baghli, L. "Contribution à la commande de la machine asynchrone, utilisation de la logique floue, des réseaux de neurones et des algorithmes génétiques" (Control of induction motors using Fuzzy logic, neural networks and genetic algorithms), PhD thesis, Henri Poincaré University, 1999. [online] Available at: https://tel. archives-ouvertes.fr/tel-00356891 [Accessed: 29 January 2020]

[2] Areed, F. G., Haikal, A. Y., Mohammed, R. H. "Adaptive neuro-fuzzy control of an induction motor", Ain Shams Engineering Journal, 1(1), pp. 71-78, 2010. https://doi.org/10.1016/j.asej.2010.09.008

[3] Zaafouri, A., Ben Regaya, C., Ben Azza, H., Chaari, A. "DSPbased adaptive backstepping using the tracking errors for high-performance sensorless speed control of induction motor drive", ISA Transactions, 60, pp. 333-347, 2016. https://doi.org/10.1016/j.isatra.2015.11.021

[4] Aichi, B., Bourahla, M., Kendouci, K., Mazari, B. "Real-time nonlinear speed control of an induction motor based on a new advanced integral backstepping approach", Transactions of the Institute of Measurement and Control, 42(2), pp. 244-258, 2020.

https://doi.org/10.1177/0142331219866545

[5] Aichi, B., Kendouci, K. "Robust and Stable Speed Control Design Using the Variable Gains Backstepping Technique for HighEfficiency Three-Phase Induction Motor Drives", In: 2020 1st International Conference on Communications, Control Systems and Signal Processing (CCSSP), EL OUED, Algeria, 2020, pp. 376-381. https://doi.org/10.1109/CCSSP49278.2020.9151709

[6] Talla, J., Leu, V. Q., Šmídl, V., Peroutka, Z. "Adaptive Speed Control of Induction Motor Drive With Inaccurate Model", IEEE Transactions on Industrial Electronics, 65(11), pp. 8532-8542, 2018. https://doi.org/10.1109/TIE.2018.2811362

[7] Ben Regaya, C., Farhani, F., Zaafouri, A., Chaari, A. "A novel adaptive control method for induction motor based on Backstepping approach using dSpace DS 1104 control board", Mechanical Systems and Signal Processing, 100, pp. 466-481, 2018. https://doi.org/10.1016/j.ymssp.2017.07.017

[8] Ammar, A., Bourek, A., Benakcha, A. "Nonlinear SVM-DTC for induction motor drive using input-output feedback linearization and high order sliding mode control", ISA Transactions, 67, pp. 428-442, 2017.

https://doi.org/10.1016/j.isatra.2017.01.010

[9] Utkin, V., Guldner, J., Shi, J. "Sliding Mode Control in ElectroMechanical Systems", CRC Press, Boca Raton, FL, USA, 2009 https://doi.org/10.1201/9781420065619

[10] Yaichi, I., Semmah, A., Wira, P., Djeriri, Y. "Super-twisting Sliding Mode Control of a Doubly-fed Induction Generator Based on the SVM Strategy", Periodica Polytechnica Electrical Engineering and Computer Science, 63(3), pp. 178-190, 2019. https://doi.org/10.3311/PPee.13726

[11] Lascu, C., Blaabjerg, F. "Super-twisting sliding mode direct torque contol of induction machine drives", In: 2014 IEEE Energy Conversion Congress and Exposition (ECCE), Pittsburgh, PA, USA, 2014, pp. 5116-5122.

https://doi.org/10.1109/ECCE.2014.6954103
[12] Jamoussi, K., Ouali, M., Chrifi-Alaoui, L., Benderradji, H., El Hajjaji, A. "Robust Sliding Mode Control Using Adaptive Switching Gain for Induction Motors", International Journal of Automation and Computing, 10(4), pp. 303-311, 2013. https://doi.org/10.1007/s11633-013-0725-x

[13] Aichi, B., Bourahla, M., Kendouci, K. "Nonlinear Speed Control of Induction Motor by the Combination of Fuzzy-Sliding-Mode and Integral-Backstepping Controllers", In: 2018 International Conference on Applied Smart Systems (ICASS), Medea, Algeria, 2018, pp. 1-6.

https://doi.org/10.1109/ICASS.2018.8652016

[14] Hamzaoui, A., Manamanni, N., Essounbouli, N., Zaytoon, J. "Switching Controllers' Synthesis: Combination of a Sliding Mode and H Infinity Control by a Fuzzy Supervisor", IFAC Proceedings Volumes, 36(6), pp. 247-252, 2003. https://doi.org/10.1016/S1474-6670(17)36439-X

[15] Barrero, F., Gonzalez, A., Torralba, A., Galvan, E., Franquelo, L. G. "Speed control of induction motors using a novel fuzzy sliding-mode structure", IEEE Transactions on Fuzzy Systems, 10(3), pp. 375-383, 2002. https://doi.org/10.1109/TFUZZ.2002.1006440

[16] Quintero-Manriquez, E., Sanchez, E. N., Harley, R. G., Li, S., Felix, R. A. "Neural Sliding Mode Control for Induction Motors Using Rapid Control Prototyping", IFAC-PapersOnLine, 50(1), pp. 9625-9630, 2017. https://doi.org/10.1016/j.ifacol.2017.08.1711

[17] Wong, L. K., Leung, F. H. F., Tam, P. K. S. "Combination of sliding mode controller and PI controller using fuzzy logic controller", In: 1998 IEEE International Conference on Fuzzy Systems Proceedings. IEEE World Congress on Computational Intelligence (Cat. No.98CH36228), Anchorage, AK, USA, 1998, pp. 296-301. https://doi.org/10.1109/FUZZY.1998.687501

[18] Essounbouli, N., Hamzaoui, A., Manamanni, N. "Fuzzy Supervisor for Combining Sliding Mode Control and $\mathrm{H} \infty$ Control", In: Bilgiç, T., De Baets, B., Kaynak, O. (eds.) Fuzzy Sets and Systems - IFSA 2003, IFSA 2003: Lecture Notes in Computer Science (Lecture Notes in Artificial Intelligence), Springer, Berlin, Heidelberg, Germany, pp. 466-473. https://doi.org/10.1007/3-540-44967-1_56

[19] Oliveira, C. M. R., Aguiar, M. L., Monteiro, J. R. B. A., Pereira, W. C. A., Paula, G. T., Almeida, T. E. P. "Vector Control of Induction Motor Using an Integral Sliding Mode Controller with Anti-windup", Journal of Control, Automation and Electrical Systems, 27(2), pp. 169-178, 2016. https://doi.org/10.1007/s40313-016-0228-4

[20] Slotine, J. J. E., Coetsee, J. A. "Adaptive sliding controller synthesis for non-linear systems", International Journal of Control, 43(6), pp. 1631-1651, 1986. https://doi.org/10.1080/00207178608933564 